\title{
Energy-Momentum Spectroscopy by Highly Parallelized Wavelength- and Angularly-Resolved Cathodoluminescence in the Scanning Electron Microscope
}

\author{
M. Bertilson ${ }^{1}$, D.J Stowe ${ }^{1}$, T Worsley ${ }^{1}$, T. Franklin ${ }^{1}$, L. Murray ${ }^{1}$, M. Hsueh ${ }^{1}$ and J.A Hunt ${ }^{1}$ \\ 1. Gatan Inc, Pleasanton, CA.
}

Researchers are actively seeking new ways to direct and enhance the radiation or absorption of lightemitting and light-harvesting devices [e.g. 1]. One highly promising approach is to make use of nanostructured surfaces or objects whose interactions with light may be modified through a change of their size, shape, or composition. Characterization of the optical properties of materials with spatial resolution better than the diffraction limit of light is required to further our understanding of light-matter interactions in nanostructured materials and devices. Thus, the technique of cathodoluminescence (CL) in the scanning electron microscope (SEM) has gained great interest due to the ability to excite optical processes with a sub-nanometer probe of (fast) electrons, correlating optical information with sample morphology, composition and crystal structure at the nanoscale [e.g. 2].

Light emission is defined by its distribution in energy (wavelength), momentum (angular), and polarization. Typically, researchers maximize the signal in one distribution by integrating over or filtering out the other two thereby eliminating information in the other two distributions; an issue that has become important in nanophotonics. Previous work [e.g. 4] demonstrated momentum and energy-momentum spectra in a CL setup. However, these experiments are limited to a single (selected) wavelength or angle respectively making a complete analysis of the wavelength and angular distributions impractical. We demonstrate an optimized approach to energy-momentum spectroscopy using angular-resolved CL measurements in the SEM. The focused electron beam excites optical transitions and the far field radiation pattern is collected by an off-axis parabolloidal mirror and coupled, via a spectrograph, to an array detector. The resultant 2D data set reveals the distribution of light - with full wavelength resolution - at up to 400 unique angles (zenith and azimuthal) simultaneously (Figure 1); the energy-momentum spectrum may be captured by scanning through the angular space. In this way, radiation is analyzed in a highly parallelized manner with high spatial, angular and wavelength resolutions (up to $10 \mathrm{~nm}, 1^{\circ}$ and 0.1 nm respectively).

In this demonstration, energy-filtered momentum spectra were reconstructed from samples using 30 energy-resolved momentum spectra; complete wavelength and angular distributions were acquired in $<150$ s. A phosphor powder film (Figure 1) was found to emit light with wavelength spectra of identical form at all angles, however, light emitted from InGaN multi-quantum wells (MQWs) exhibited significant variance (Figure 2a and 2b). The MQWs were situated $\sim 100 \mathrm{~nm}$ below the surface of a $4.9 \mu \mathrm{m}$ thick GaN film on sapphire substrate. The strong variance of the wavelength distribution as a function of angle could be ascribed to interference in the far field between light emitted from the excitation point and light reflected at the rear surface of the sample. Simulation was found to agree with experiment (Figure 2c and $2 \mathrm{~d}$ ) assuming absorption and reflectivity coefficients of $\mathrm{GaN}$ on (0001) $\alpha-\mathrm{Al}_{2} \mathrm{O}_{3}$ from [4] and using Fresnel's equations and basic thin-film interference with a degree of coherence of 0.35 .

As researchers seek new ways to direct and enhance the radiation from light emitting and harvesting devices, such streamlined methods to collect energy-momentum spectra, as demonstrated here, will become increasingly important. 
References:

[1] Q Jiao et al, Nanoscale Res. Lett. 11 (2016), p. 340.

[2] M Knight et al, Nano Lett., 12 (2012), p. 6000.

[3] N Yamamoto in "The Transmission Electron Microscope", Ed. K Maaz, (In Tech).

[4] A Barker Jr. and M. Ilegems. Phys. Rev. B 7 (1973), p. 743.

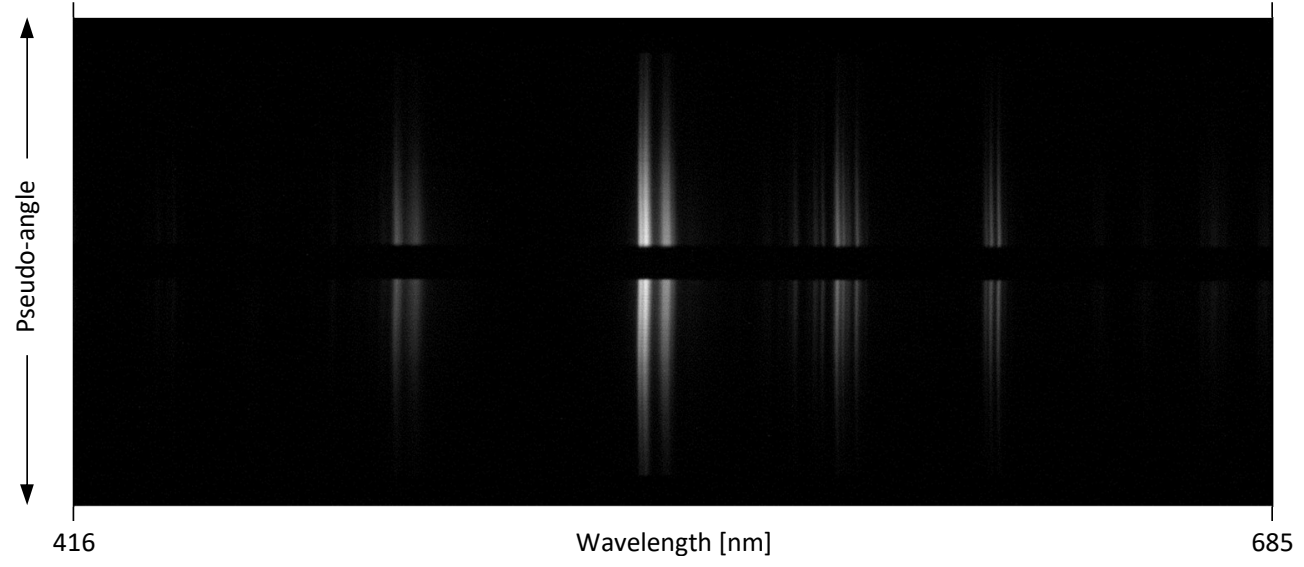

Figure 1. Energy-resolved momentum spectrum from a phosphor particle collected in a cathodoluminescence (CL) experimental setup. The angular distribution exhibits isotropic color (wavelength invariance) at all angles.

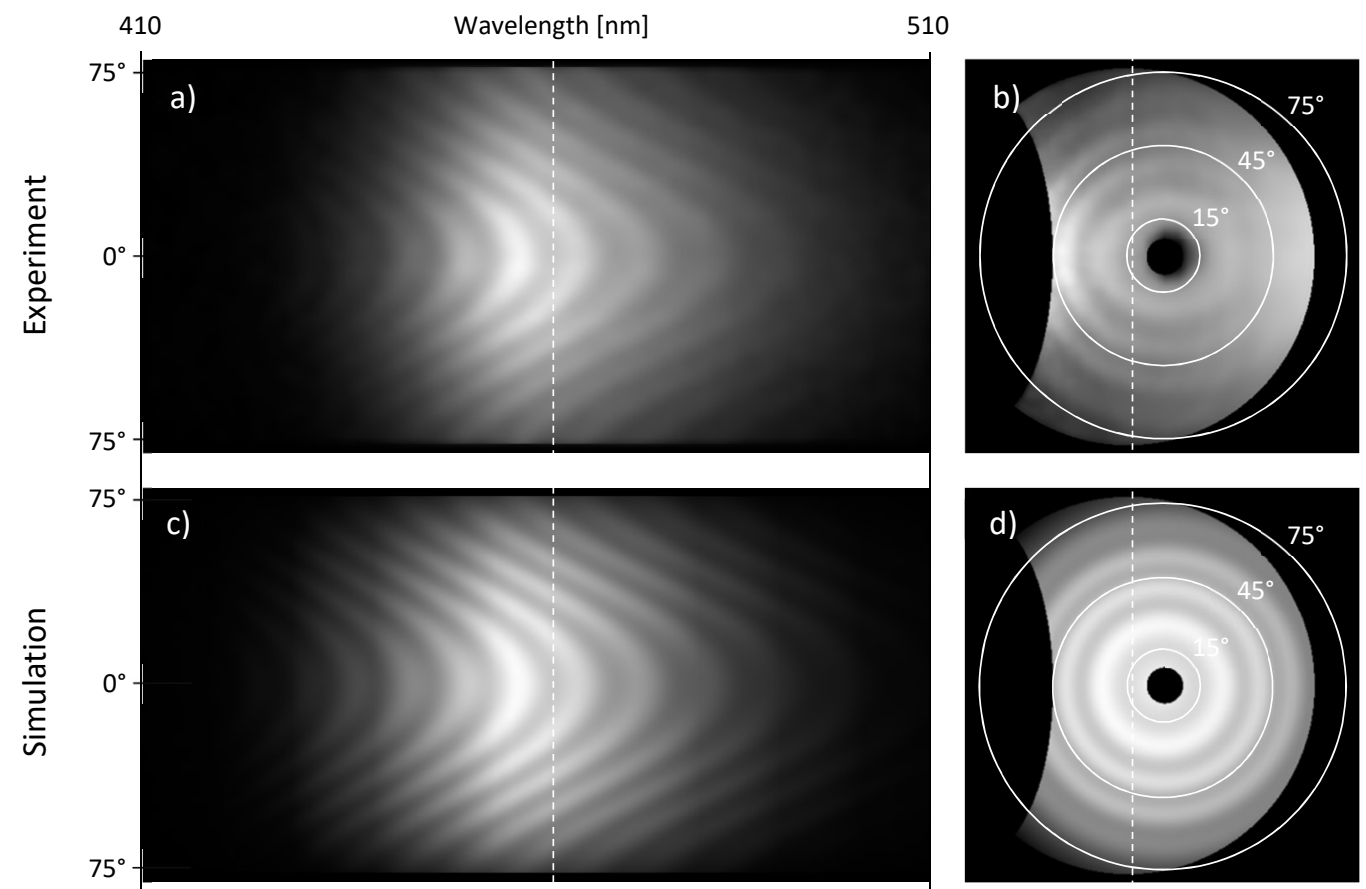

Figure 2. Slices extracted for the reconstructed 3D energy-momentum CL spectrum of InGaN multiquantum wells in GaN film demonstrating strong angular dependence of the wavelength distribution: a) and c) are momentum-filtered energy spectra through the reconstruction at position indicated with the dotted line in b) and d); b) and d) are energy-filtered momentum spectra from the same data sets respectively displaying the angular distribution of light of wavelength at $\sim 462 \mathrm{~nm}$. 